\title{
Uniqueness of meromorphic functions sharing two values
}

Yin-Hong $\mathrm{Cao}^{1 *}$ and Xiao-Bin Zhang ${ }^{2}$

* Correspondence: yhcao82@yahoo.cn

${ }^{1}$ School of Mathematics, Shandong University, Jinan, Shandong 250100, People's Republic of China Full list of author information is available at the end of the article

\section{Abstract}

In this article, we shall study the uniqueness problems on meromorphic functions sharing nonzero finite value or having fixed points. Our results extend the corresponding results of Fang and Hua, Yang and Hua, and Fang and Qiu. MSC 2010: 30D35, 30D30.

Keywords: uniqueness, meromorphic function, sharing value, fixed point

\section{Introduction and main results}

Let $\mathbb{C}$ denote the complex plane and $f$ be a nonconstant meromorphic function on $\mathbb{C}$. We assume the reader is familiar with the standard notion used in the Nevanlinna value distribution theory such as $T(r, f), m(r, f), N(r, f)$ (see, e.g., [1-4]), and $S(r, f)$ denotes any quantity that satisfies the condition $S(r, f)=o(T(r, f))$ as $r \rightarrow \infty$ outside of a possible exceptional set of finite linear measure. A meromorphic function $a$ is called a small function with respect to $f$, provided that $T(r, a)=S(r, f)$.

Let $f$ and $g$ be two nonconstant meromorphic functions. Let $a$ be a small function of $f$ and $g$. We say that $f, g$ share $a$ counting multiplicities (CM) if $f-a, g-a$ have the same zeros with the same multiplicities and we say that $f, g$ share $a$ ignoring multiplicities (IM) if we do not consider the multiplicities. We denote $\bar{N}_{0}(r, \infty)$ the reduced counting function of the common poles of $f$ and $g$. If $\bar{N}(r, f)-\bar{N}_{0}(r, \infty)=S(r, f)$, and $\bar{N}(r, g)-\bar{N}_{0}(r, \infty)=S(r, g)$, we say that $f$ and $g$ share $\infty$ "IM". We denote by $N_{k}\left(r, \frac{1}{f-a}\right)\left(\operatorname{or} \bar{N}_{k}\left(r, \frac{1}{f-a}\right)\right)$ the counting function for zeros of $f-a$ with multiplicity $\leq k$ (IM), and by $N_{(k}\left(r, \frac{1}{f-a}\right)\left(\operatorname{or} \bar{N}_{k}\left(r, \frac{1}{f-a}\right)\right)$ the counting function for zeros of $f-a$ with multiplicity $\leq k$ (IM). Moreover, we set $N_{k}\left(r, \frac{1}{f-a}\right)=\bar{N}\left(r, \frac{1}{f-a}\right)+\bar{N}_{2}\left(r, \frac{1}{f-a}\right)+\bar{N}_{3}\left(r, \frac{1}{f-a}\right)+\cdots+\bar{N}_{k}\left(r, \frac{1}{f-a}\right)$.

We say that a finite value $z_{0}$ is called a fixed point of $f$ if $f\left(z_{0}\right)=z_{0}$ or $z_{0}$ is a zero of $f(z)-z$.

The following theorem in the value distribution theory is well known $[5,6]$.

Theorem A. Let $f$ be a transcendental meromorphic function, $n \leq 1$ a positive integer. Then $f^{n} \dot{f}^{\prime}=1$ has infinitely many solutions.

Fang and Hua [7], Yang and Hua [8] got a unicity theorem, respectively, corresponding to Theorem A.

\section{Springer}

(C) 2012 Yin-Hong and Zhang; licensee Springer. This is an Open Access article distributed under the terms of the Creative Commons Attribution License (http://creativecommons.org/licenses/by/2.0), which permits unrestricted use, distribution, and reproduction in any medium, provided the original work is properly cited. 
Theorem B. Let $f$ and $g$ be two nonconstant entire (meromorphic) functions, $n \leq 6(n$ $\leq 11)$ be a positive integer. If $f^{n} f^{\prime}$ and $g^{n} g^{\prime}$ share $1 C M$, then either $f(z)=c_{1} e^{c z}, g(z)=$ $c_{2} e^{-c z}$, where $c_{1}, c_{2}$ and c are three constants satisfying $4\left(c_{1} c_{2}\right)^{n+1} c^{2}=-1$, or $f \equiv \operatorname{tg}$ for a constant $t$ such that $t^{n+1}=1$.

Considering the uniqueness question of entire or meromorphic functions having fixed points, Fang and Qiu [9] obtained the following result.

Theorem C. Let $f$ and $g$ be two nonconstant meromorphic (entire) functions, $n \leq 11$ ( $n$ $\leq 6)$ a positive integer. If $f^{n} f^{\prime}$ and $g^{n} g^{\prime}$ share $z C M$, then either $f(z)=c_{1} e^{c z^{2}}$, $g(z)=c_{2} e^{-c z^{2}}$, where $c_{1}, c_{2}$, and $c$ are three constants satisfying $4\left(c_{1} c_{2}\right)^{n+1} c^{2}=-1$, or $f \equiv$ tg for a constant $t$ such that $t^{n+1}=1$.

For more results in such directions, we refer the readers to [7-24].

We recall the following result by $\mathrm{Xu}$ et al. [25] or Zhang and $\mathrm{Li}$ [26], respectively.

Theorem D. Let $f$ be a transcendental meromorphic function, $n(\leq 2), k$ be two positive integers. Then $f^{n} f^{(k)}$ takes every finite nonzero value infinitely many times or has infinitely many fixed points.

Corresponding to Theorem D, one may ask, what can be said about the relationship between two meromorphic functions $f$ and $g$, if $f^{n} f^{(k)}$ and $g^{n} g^{(k)}$ have the same fixed points or share one nonzero complex number, where $n$ and $k$ are positive numbers? In this direction, we will prove:

Theorem 1.1. Let $f$ and $g$ be two transcendental meromorphic functions, whose zeros are of multiplicities at least $k$, where $k$ is a positive integer. Let $n>\max \{2 k-1, k+4 / k+$ 4) be a positive integer. If $f^{n} f^{(k)}$ and $g^{n} g^{(k)}$ share $z C M$, $f$ and $g$ share $\infty I M$, one of the following two conclusions holds:

(i) $f^{n} f^{(k)}=g^{n} g^{(k)}$;

(ii) $f(z)=c_{1} e^{c z^{2}}, g(z)=c_{2} e^{-c z^{2}}$, where $c_{1}, c_{2}$, and $c$ are constants such that $4\left(c_{1} c_{2}\right)^{n+1} c^{2}=-1$.

Theorem 1.2. Let $f$ and $g$ be two nonconstant meromorphic functions, whose zeros are of multiplicities at least $k$, where $k$ is a positive integer. Let $n>\max \{2 k-1, k+4 / k+4\}$ be a positive integer. If $f^{n} f^{(k)}$ and $g^{n} g^{(k)}$ share $1 C M, f$ and $g$ share $\infty I M$, one of the following two conclusions holds:

(i) $f^{n} f^{(k)}=g^{n} g^{(k)}$;

(ii) $f(z)=c_{3} e^{d z}, g(z)=c_{4} e^{-d z}$, where $c_{3}, c_{4}$, and $d$ are constants such that $(-1)^{k}\left(c_{3} c_{4}\right)^{n+1}$ $d^{2 k}=1$.

Remark 1.1. Theorems 1.1 and 1.2 are also true of entire functions when $n>4 / k+2$ be a positive integer.

Theorem 1.3. Let $f$ and $g$ be two nonconstant meromorphic functions, whose zeros are of multiplicities at least $k+1$, where $k$ is a positive integer with $1 \leq k \leq 5$. Let $n \leq 10$ be a positive integer. If $f^{n} f^{(k)}$ and $g^{n} g^{(k)}$ share $1 C M, f^{(k)}$ and $g^{(k)}$ share $1 C M, f$ and $g$ share $\infty I M$, one of the following two conclusions holds:

(i) $f \equiv$ tg for a constant $t$ such that $t^{n+1}=1$;

(ii) $f(z)=c_{3} e^{d z}, g(z)=c_{4} e^{-d z}$, where $c_{3}, c_{4}$ and $d$ are constants such that $(-1)^{k}\left(c_{3} c_{4}\right)^{n+1} d^{2 k}=1$.

\section{Preliminary lemmas}

Lemma 2.1. [3] Let $f$ be a nonconstant meromorphic function, and let $k$ be a positive integer. Suppose that $f^{(k)} \neq 0$, then 


$$
N\left(r, \frac{1}{f^{(k)}}\right) \leq N\left(r, \frac{1}{f}\right)+k \bar{N}(r, f)+S(r, f) .
$$

By using the similar method of Yang and Hua [8], we can prove the following lemma.

Lemma 2.2. Let $f$ and $g$ be two nonconstant meromorphic functions, a be a finite nonzero constant. If $f$ and $g$ share a CM and $\infty$ "IM", one of the following cases holds:

(i) $T(r, f) \leq N_{2}(r, 1 / f)+N_{2}(r, 1 / g)+3 \bar{N}(r, f)+S(r, f)+S(r, g)$, the same inequality holding for $T(r, g)$;

(ii) $f g \equiv a^{2}$; (iii) $f \equiv g$.

Lemma 2.3. [1, Theorem 3.10] Suppose that $f$ is a nonconstant meromorphic function, $k \leq 2$ is an integer. If

$$
N(r, f)+N(r, 1 / f)+N\left(r, 1 / f^{(k)}\right)=S\left(r, f^{\prime} / f\right),
$$

Then $f(z)=e^{a z+b}$, where $a \neq 0, b$ are constants.

Lemma 2.4. [27] Let $f$ and $g$ be nonconstant meromorphic functions. Suppose that $f$ and $g$ share the values $O$ and $\infty C M, f^{(k)}$ and $g^{(k)}$ share the value $0 C M$ for $k=1,2,3$, $4,5,6$. Then $f$ and $g$ satisfy one of the following cases:

(i) $f=$ tg, where $t(\neq 0)$ is a constant.

(ii) $f(z)=e^{a z+b}, g(z)=e^{c z}+^{d}$, where $a, b, c$, and d are constants with $a c \neq 0$.

(iii) $f(z)=\frac{a}{1-b e^{\alpha(z)}}, g(z)=\frac{a}{e^{-\alpha(z)}-b}$, where $a, b$ are nonzero consants, and $\alpha(z)$ is a nonconstant entire function.

(iv) $f(z)=a\left(1-b e^{c z}\right), g(z)=d\left(e^{-c z}-b\right)$, where $a, b, c$, and $d$ are nonzero constants.

To prove Theorems 1.1 and 1.2, we also need the following results.

Lemma 2.5. Let $f, g$ be two nonconstant meromorphic functions, whose zeros are of multiplicities at least $k$, where $k$ is a positive integer. Let $n>2 k-1$ be a positive integer. If $f^{n} f^{(k)} g^{n} g^{(k)}=z^{2}$, f and $g$ share $\infty I M$, then $f(z)=c_{1} e^{c z^{2}} g(z)=c_{2} e^{-c z^{2}}$, where $c_{1}, c_{2}$, and $c$ are constants such that $4\left(c_{1} c_{2}\right)^{n+1} c^{2}=-1$.

Lemma 2.6. Let $f, g$ be two nonconstant meromorphic functions, whose zeros are of multiplicities at least $k$, where $k$ is a positive integer. Let $n>2 k-1$ be a positive integer. If $f^{n} f^{(k)} g^{n} g^{(k)}=1, f$ and $g$ share $\infty I M$, then $f(z)=c_{3} e^{d z}, g(z)=c_{4} e^{-d z}$, where $c_{3}, c_{4}$, and $d$ are constants such that $(-1)^{k}\left(c_{3} c_{4}\right)^{n+1} d^{2 k}=1$.

\section{Proofs of Lemmas 2.5 and 2.6}

\subsection{Proof of Lemma 2.5}

Since $f$ and $g$ share $\infty \mathrm{IM}$, we have from

$$
f^{n} f^{(k)} g^{n} g^{(k)}=z^{2}
$$

that $f$ and $g$ are entire functions.

Suppose that $f$ has a zero $z_{0}$ of multiplicity $p \leq k$, then $z_{0}$ is a zero of $f^{n} f^{(k)}$ with multiplicity $n p+p-k \leq 2 k^{2}$. In view of (3.1), we get $k=1, n=2$, and $z_{0}=0$.

Moreover, $g$ has no zero. Therefore,

$$
f(z)=z e^{\alpha_{1}(z)}, g(z)=e^{\beta_{1}(z)},
$$


where $\alpha_{1}(z), \beta_{1}(z)$ are nonconstant entire functions. So from 3.2, we get

$$
z^{2}\left(1+z \alpha_{1}^{\prime}(z)\right) \beta_{1}^{\prime} e^{3\left(\alpha_{1}(z)+\beta_{1}(z)\right)}=z^{2},
$$

namely

$$
\left(1+z \alpha_{1}^{\prime}(z)\right) \beta_{1}^{\prime} e^{3\left(\alpha_{1}(z)+\beta_{1}(z)\right)}=1,
$$

which is impossible since $\alpha_{1}$ and $\beta_{1}$ are non-constant entire functions $\left(\alpha^{\prime}{ }_{1} \equiv 0, \beta^{\prime}{ }_{1} \equiv 0\right)$. Thus $f$ has no zero. similarly, we get that $g$ has no zero. So, we have

$$
f(z)=e^{\alpha(z)}, g(z)=e^{\beta(z)},
$$

where $\alpha(z), \beta(z)$ are nonconstant entire functions. Then

$$
T\left(r, \frac{f^{\prime}}{f}\right)=T\left(r, \alpha^{\prime}\right)
$$

We claim that $\alpha+\beta \equiv C$, where $C$ is a constant.

Let $F=f^{n} f^{(k)}, G=g^{n} g^{(k)}$. Then we have

$$
\begin{aligned}
n T(r, f) & =T\left(r, f^{n}\right)=T\left(r, \frac{F}{f^{(k)}}\right) \\
& \leq T(r, F)+T\left(r, f^{(k)}\right)+S(r, f) \leq T(r, F)+T(r, f)+S(r, f),
\end{aligned}
$$

We obtain from (3.7) that

$$
T(r, f)=O(T(r, F))
$$

as $r \in E$ and $r \rightarrow \infty$, where $E \subset(0,+\infty)$ is some subset of finite linear measure. Note that

$$
\begin{aligned}
T(r, F) & =T\left(r, f^{n} f^{(k)}\right)+n T(r, f)+T\left(r, f^{(k)}\right)+S(r, f) \\
& \leq(n+1) T(r, f)+S(r, f),
\end{aligned}
$$

We obtain from (3.9) that

$$
T(r, F)=O(T(r, f)),
$$

as $r \in E$ and $r \rightarrow \infty$, where $E \subset(0,+\infty)$ is some subset of finite linear measure.

Thus from (3.8), (3.10) and the standard reasoning of removing exceptional set (see [2, Lemma 1.1.1]) we deduce $\sigma(f)=\sigma(F)$. Similarly, we have $\sigma(g)=\sigma(G)$. It follows from (3.1) that $\sigma(F)=\sigma(G)$, we get $\sigma(f)=\sigma(g)$.

We deduce that either both $\alpha$ and $\beta$ are transcendental functions or both $\alpha$ and $\beta$ are polynomials. Moreover, we have

$$
N\left(r, 1 / f^{(k)}\right) \leq N\left(r, 1 / z^{2}\right)=O(\log r) .
$$

From this and (3.5) we get

$$
N(r, f)+N(r, 1 / f)+N\left(r, 1 / f^{(k)}\right)=O(\log r) .
$$


If $k \leq 2$, then it follows from (3.6) and Lemma 2.3 that $\alpha$ is a polynomial, and so $\alpha$ is a nonconstant polynomial. Similarly, we can deduce that $\beta$ is also a nonconstant polynomial.

We deduce from (3.5) that

$$
f^{(k)}=\left[\left(\alpha^{\prime}\right)^{k}+P_{k-1}\left(\alpha^{\prime}\right)\right] e^{\alpha}, g^{(k)}=\left[\left(\beta^{\prime}\right)^{k}+Q_{k-1}\left(\beta^{\prime}\right)\right] e^{\beta},
$$

where $P_{k-1}\left(\alpha^{\prime}\right)$ and $Q_{k-1}\left(\beta^{\prime}\right)$ are differential polynomials in $\alpha^{\prime}$ and $\beta^{\prime}$ of degree at most $k-1$, respectively. Thus, we obtain

$$
\left[\left(\alpha^{\prime}\right)^{k}+P_{k-1}\left(\alpha^{\prime}\right)\right]\left[\left(\beta^{\prime}\right)^{k}+Q_{k-1}\left(\beta^{\prime}\right)\right] e^{(n+1)(\alpha+\beta)}=z^{2} .
$$

We deduce from (3.11) that $\alpha(z)+\beta(z) \equiv C$ for a constant $C$.

If $k=1$, from (3.1) and (3.5) we get

$$
\alpha^{\prime} \beta^{\prime} e^{(n+1)(\alpha+\beta)}=z^{2} .
$$

Next, we let $\alpha+\beta=\gamma$ and suppose that $\alpha, \beta$ are transcendental entire functions.

If $\gamma$ is a constant, then $\alpha^{\prime}+\beta^{\prime}=0$, and from (3.12) we have $\alpha^{\prime} \beta^{\prime} e^{(n+1) \gamma}=-\alpha^{\prime 2} e^{(n+1) \gamma}=z^{2}$ which implies that $\alpha^{\prime}$ is a nonconstant polynomial of degree $\operatorname{deg}\left(\alpha^{\prime}\right)=1$. This together with $\alpha^{\prime}+\beta^{\prime}=0$ implies that $\beta^{\prime}$ is also a nonconstant polynomial of $\operatorname{degree} \operatorname{deg}\left(\beta^{\prime}\right)=1$.

If $\gamma$ is not a constant, then (3.12) implies that

$$
\alpha^{\prime}\left(\gamma^{\prime}-\alpha^{\prime}\right) e^{(n+1) \gamma}=z^{2} .
$$

Since $T\left(r, \gamma^{\prime}\right)=m\left(r, \gamma^{\prime}\right) \leq m\left(r, \frac{\left(e^{(n+1) \gamma}\right)^{\prime}}{e^{(n+1) \gamma}}\right)+O(1)=S\left(r, e^{(n+1) \gamma}\right)$. Thus

implies that

$$
\begin{aligned}
T\left(r, e^{(n+1) \gamma}\right) & \leq T\left(r, \frac{z^{2}}{\alpha^{\prime}\left(\gamma^{\prime}-\alpha^{\prime}\right)}\right)+O(1) \\
& \leq(2+o(1)) T\left(r, \alpha^{\prime}\right)+S\left(r, e^{n \gamma}\right),
\end{aligned}
$$

which implies that

$$
T\left(r, e^{(n+1) \gamma}\right)=O\left(T\left(r, \alpha^{\prime}\right)\right),
$$

Similarly, we have

$$
T\left(r, \alpha^{\prime}\right)=O\left(T\left(r, e^{(n+1) \gamma}\right)\right) .
$$

Thus $T\left(r, \gamma^{\prime}\right)=S\left(r, e^{(n+1) \gamma}\right)=S\left(r, \alpha^{\prime}\right)$.

In view of (3.13) and by the second fundamental theorem for small functions, we get

$$
T\left(r, \alpha^{\prime}\right) \leq \bar{N}\left(r, \frac{1}{\alpha^{\prime}}\right)+\bar{N}\left(r, \frac{1}{\alpha^{\prime}-\gamma^{\prime}}\right)+S\left(r, \alpha^{\prime}\right) \leq O(\log r)+S\left(r, \alpha^{\prime}\right) .
$$

Thus $\alpha^{\prime}$ is a polynomial, which contradicts that $\alpha$ is a transcendental entire function. Thus $\alpha$ and $\beta$ are both polynomials and $\alpha(z)+\beta(z) \equiv C$ for a constant $C$.

Hence, from (3.11) we get

$$
(-1)^{k}\left(\alpha^{\prime}\right)^{2 k}=z^{2}+\tilde{P}_{2 k-1}\left(\alpha^{\prime}\right)
$$


where $\tilde{P}_{2 k-1}$ is a differential polynomial in $\alpha^{\prime}$ of degree at most $2 k-1$. From (3.14) we have

$$
2 k T\left(r, \alpha^{\prime}\right)=2 \log r+S\left(r, \alpha^{\prime}\right) .
$$

From (3.15) we can see that $\alpha^{\prime}$ is a nonconstant polynomial of degree 1 and that $k=1$.

By induction we get

$$
\begin{aligned}
& \alpha^{\prime}+\beta^{\prime}=0, \\
& e^{(n+1) C} \alpha^{\prime} \beta^{\prime}=z^{2} .
\end{aligned}
$$

By computation we get

$$
\alpha^{\prime}=l_{1} z, \quad \beta^{\prime}=-l_{2} z,
$$

Hence

$$
\alpha=c z^{2}+l_{3}, \quad \beta=-c z^{2}+l_{4},
$$

We can rewrite $f$ and $g$ as

$$
f=c_{1} e^{c z^{2}}, \quad g=c_{2} e^{-c z^{2}},
$$

where $c_{1}, c_{2}$, and $c$ are constants such that $4\left(c_{1} c_{2}\right)^{n+1} c^{2}=-1$.

This completes the proof of Lemma 2.5 .

\subsection{Proof of Lemma 2.6}

By the same reasons as in Lemma 2.5, we get

$$
f(z)=e^{\alpha(z)}, \quad g(z)=e^{\beta(z)},
$$

and

$$
\left(\alpha^{\prime}\right)^{2 k}=1+\tilde{P}_{2 k-1}\left(\alpha^{\prime}\right)
$$

where $\tilde{P}_{2 k-1}$ is a differential polynomial in $\alpha^{\prime}$ of degree at most $2 k-1$. From (3.19) we have

$$
2 k T\left(r, \alpha^{\prime}\right) \leq(2 k-1) T\left(r, \alpha^{\prime}\right)+S\left(r, \alpha^{\prime}\right),
$$

which implies that $\alpha^{\prime}$ is a nonzero constant. Thus $\alpha=d z+l_{5}, \beta=-d z+l_{6}$. By (3.18), rewrite $f$ and $g$ as

$$
f=c_{3} e^{d z}, \quad g=c_{4} e^{-d z},
$$

where $c_{3}, c_{4}$ and $d$ are constants such that $(-1)^{k}\left(c_{3} c_{4}\right)^{n+1} d^{2 k}=1$.

This completes the proof of Lemma 2.6.

\section{Proof of Theorem 1.1}

Let $F=f^{n} f^{(k)}, G=g^{n} g^{(k)}, F^{*}(z)=F(z) / z, G^{*}(z)=G(z) / z$. Note that $f$ and $g$ are transcendental, so $z$ is a small function with respect to both $F$ and $G$. Then $F^{*}$ and $G^{*}$ share $1 \mathrm{CM}$ and $\infty$ "IM". By Lemma 2.2, we consider three cases. 
Case 1. Suppose that

$$
T\left(r, F^{*}\right) \leq N_{2}\left(r, 1 / F^{*}\right)+N_{2}\left(r, 1 / G^{*}\right)+3 \bar{N}(r, f)+S(r, f)+S(r, g) .
$$

We deduce from (4.1) that

$$
T(r, F) \leq N_{2}(r, 1 / F)+N_{2}(r, 1 / G)+3 \bar{N}(r, f)+S(r, f)+S(r, g) .
$$

Obviously,

$$
N(r, F)=(n+1) N(r, f)+k \bar{N}(r, f)+S(r, f) .
$$

Since

$$
\begin{aligned}
n m(r, f) & =m\left(r, F / f^{(k)}\right) \leq m(r, F)+m\left(r, 1 / f^{(k)}\right)+S(r, f) \\
& =m(r, F)+T\left(r, f^{(k)}\right)-N\left(r, 1 / f^{(k)}\right)+S(r, f) \\
& \leq m(r, F)+T(r, f)+k \bar{N}(r, f)-N\left(r, 1 / f^{(k)}\right)+S(r, f) .
\end{aligned}
$$

It follows from (4.2), (4.3), and Lemma 2.1 that

$$
\begin{aligned}
(n-1) T(r, f) \leq & T(r, F)-N(r, f)-N\left(r, 1 / f^{(k)}\right)+S(r, f) \\
\leq & N_{2}(r, 1 / F)+N_{2}(r, 1 / G)+3 \bar{N}(r, f) \\
& -N(r, f)-N\left(r, 1 / f^{(k)}\right)+S(r, f)+S(r, g) \\
\leq & N_{2}\left(r, 1 / f^{n}\right)+N_{2}\left(r, 1 / f^{(k)}\right)+N_{2}\left(r, 1 / g^{n}\right)+N_{2}\left(r, 1 / g^{(k)}\right) \\
& +3 N(r, f)-N(r, f)-N\left(r, 1 / f^{(k)}\right)+S(r, f)+S(r, g) \\
\leq & 2 \bar{N}(r, 1 / f)+2 \bar{N}(r, 1 / g)+N\left(r, 1 / f^{(k)}\right)+N\left(r, 1 / g^{(k)}\right) \\
& +2 N(r, f)-N\left(r, 1 / f^{(k)}\right)+S(r, f)+S(r, g) \\
\leq & \frac{2}{k} N(r, 1 / f)+\frac{2}{k} N(r, 1 / g)+N(r, 1 / g)+k \bar{N}(r, g) \\
& +2 N(r, g)+S(r, f)+S(r, g) \\
\leq & \frac{2}{k}(T(r, f)+T(r, g))+(k+3) T(r, g)+S(r, f)+S(r, g) .
\end{aligned}
$$

Similarly, we have

$$
(n-1) T(r, g) \leq \frac{2}{k}(T(r, f)+T(r, g))+(k+3) T(r, f)+S(r, f)+S(r, g) .
$$

Combining the above two inequalities gives

$$
(n-1)(T(r, f)+T(r, g)) \leq\left(\frac{4}{k}+k+3\right)(T(r, f)+T(r, g))+S(r, f)+S(r, g) .
$$

Note that $n>k+4 / k+4$, we get a contradiction from (4.4).

Case 2. Suppose that $f^{n} f^{(k)} g^{n} g^{(k)}=z^{2}$. Then, by Lemma 2.5, we get conclusion (ii).

Case 3. Suppose that $f^{n} f^{(k)}=g^{n} g^{(k)}$. Then we have the conclusion (i) of Theorem 1.1. This completes the proof of Theorem 1.1.

By Lemma 2.6, using the same argument as in the proof of Theorem 1.1, we can prove Theorem 1.2. 


\section{Proof of Theorem 1.3}

Since $n \leq 10$, we have $n>\max \{2 k-1, k+4 / k+4\}$ for $1 \leq k \leq 5$. By Theorem 1.2, one of the following two conclusions holds:

(i) $f^{n} f^{(k)}=g^{n} g^{(k)}$;

(ii) $f(z)=c_{3} e^{d z}, g(z)=c_{4} e^{-d z}$, where $c_{3}, c_{4}$, and $d$ are constants such that $(-1)^{k}\left(c_{3} c_{4}\right)^{n+1} d^{2 k}=1$. We only need to consider the case (1). Namely

$$
f^{n} f^{(k)}=g^{n} g^{(k)} .
$$

Let

$$
h=g / f .
$$

We claim that $h$ is a nonzero constant.

If $h$ is not a constant, we claim that

$$
h=e^{\gamma},
$$

where $\gamma$ is a nonconstant entire function. Thus we need to prove that

(a) $f$ and $g$ share $\infty \mathrm{CM}$;

(b) $f$ and $g$ share 0 CM.

We prove the above two conclusions step-by-step.

Step 1. We prove (a).

Note that $f$ and $g$ share $\infty$ IM. Suppose that $z_{0}$ is a pole of $f$ with multiplicity $p$, a pole of $g$ with multiplicity $q$. From (5.1) we have

$$
n p+p+k=n q+q+k,
$$

namely

$$
(n+1)(p-q)=0,
$$

which implies $p=q$, thus $f$ and $g$ share $\infty \mathrm{CM}$, we get (a).

Step 2. We prove (b).

From (5.1) and the assumptions that $f^{n} f^{(k)}$ and $g^{n} g^{(k)}$ share $1 \mathrm{CM}, f^{(k)}$ and $g^{(k)}$ share

$1 \mathrm{CM}, f$ and $g$ share $\infty \mathrm{IM}$, we can deduce $f$ and $g$ share 0 IM.

Suppose that $z_{2}$ is a zero of $f$ with multiplicity $m_{1}$, a zero of $g$ with multiplicity $m_{2}$. Then we have

$$
n m_{1}+m_{1}-k=n m_{2}+m_{2}-k,
$$

which implies $m_{1}=m_{2}$. Thus $f$ and $g$ share $0 \mathrm{CM}$, and we get (b). Therefore, (5.3) holds. Moreover, from (5.1) we get

$$
f^{(k)} / g^{(k)}=e^{n \gamma} .
$$

Thus $f^{(k)}$ and $g^{(k)}$ share 0 and $\infty$ CM. By Lemma 2.4 and note that $h$ is not a constant, we consider three cases.

Case 1. $f(z)=e^{a z+b}, g(z)=e^{c z}+{ }^{d}$, where $a, b, c$, and $d$ are constants with $a c \neq 0$. We deduce from (5.1) that

$$
(a / c)^{k} e^{(n+1)(b-d)} e^{(n+1)(a-c) z}=1,
$$


which implies $a=c$, thus $f=t_{1} g$, where $t_{1}$ is a nonzero constant, which is a contradiction since $h$ is not a constant. Case 1 has been ruled out.

Case 2. $f(z)=\frac{a}{1-b e^{\alpha(z)}}, g(z)=\frac{a}{e^{-\alpha(z)}-b}$, where $a, b$ are nonzero constants, and $\alpha(z)$ is a nonconstant entire function. Thus we have $h=g / f=e^{\alpha(z)}$. From (5.1) it is easy to obtain

$$
S(r, f)=S(r, g) .
$$

Moreover, we have from the expressions of $f$ and $g$ that

$$
T(r, f)=T\left(r, e^{\alpha(z)}\right)+O(1)=T(r, h)+S(r, h), \quad T(r, g)=T(r, h)+S(r, h) .
$$

Combining (5.9) and (5.10) gives

$$
S(r, f)=S(r, g)=S(r, h) \text {. }
$$

From (5.1) we have

$$
h^{n+1}=\frac{g f^{(k)}}{f g^{(k)}} .
$$

Note that $f$ and $g$ have no zero. By the first fundamental theorem and the lemma of logarithmic derivative, we deduce from (5.12) that

$$
\begin{aligned}
(n+1) T(r, h) & =T\left(r, h^{n+1}\right)=T\left(r, \frac{g f^{(k)}}{f g^{(k)}}\right) \leq T\left(r, \frac{f^{(k)}}{f}\right)+T\left(r, \frac{g^{(k)}}{g}\right)+O(1) \\
& =m\left(r, \frac{f^{(k)}}{f}\right)+N\left(r, \frac{f^{(k)}}{f}\right)+m\left(r, \frac{g^{(k)}}{g}\right)+N\left(r, \frac{g^{(k)}}{g}\right)+O(1) \\
& \leq k \bar{N}(r, f)+k \bar{N}(r, g)+S(r, f)+S(r, g) \\
& \leq k T(r, f)+k T(r, g)+S(r, f)+S(r, g) \\
& \leq 2 k T(r, h)+S(r, h) \leq 10 T(r, h)+S(r, h),
\end{aligned}
$$

which is a contradiction since $n \leq 11$. Case 2 has been ruled out.

Case 3. $f(z)=a\left(1-b e^{c z}\right), g(z)=d\left(e^{-c z}-b\right)$, where $a, b, c$, and $d$ are nonzero constants.

Then all zeros of $f$ and $g$ are simple, which contradicts our assumption. Case 3 has been ruled out.

Therefore, $h$ is a constant. Since $g$ is not a constant, we have $h \neq 0$. Let $t=1 / h$, and we have $f \equiv t g$, we deduce from (5.1) that $t^{n+1}=1$. This completes the proof of Theorem 1.3.

\section{Conjecture}

In this section, we pose the following

Conjecture: Let $f$ and $g$ be two nonconstant meromorphic functions, whose zeros are of multiplicities at least $k$, where $k$ is a positive integer. Let $n>\max \{2 k-1, k+4 / k+5\}$ be a positive integer. If $f^{n} f^{(k)}$ and $g^{n} g^{(k)}$ share $1 \mathrm{CM}$, then one of the following two conclusions holds:

(i) $f=\operatorname{tg}$ for a constant $t$ such that $t^{n+1}=1$;

(ii) $f(z)=c_{3} e^{d z}, g(z)=c_{4} e^{-d z}$, where $c_{3}, c_{4}$ and $d$ are constants such that $(-1)^{k}\left(c_{3} c_{4}\right)^{n+1} d^{2 k}=1$. 


\section{Acknowledgements}

The authors thank the referees for their careful reading and valuable comments to improve the article. This study was partly supported by the NNSF of China (No. 11171184). X-BZ was supported by the Scientific Research Foundation of CAUC, China (Grant No. 2011QD10X)

\section{Author details}

${ }^{1}$ School of Mathematics, Shandong University, Jinan, Shandong 250100, People's Republic of China ${ }^{2}$ School of Science, Civil Aviation University of China, Tianjin 300300, People's Republic of China

\section{Authors' contributions}

$\mathrm{YH}$ completed the main part of this article, $\mathrm{YH}$ and $\mathrm{XB}$ corrected the main theorems. All the authors read and approved the final manuscript.

\section{Competing interests}

The authors declare that they have no competing interests.

Received: 30 December 2011 Accepted: 24 April 2012 Published: 24 April 2012

\section{References}

1. Hayman, WK: Meromorphic Functions. Oxford University Press, London. (1964)

2. Laine, I: Nevanlinna Theory and Complex Differential Equations. Walter de Gruyter, Berlin (1993)

3. Yang, CC, Yi, HX: Uniqueness Theory of Meromorphic Functions. Kluwer Academic Publisher, Dordrecht. (2003)

4. Yang, L: Value Distribution Theory. Springer-Verlag, Berlin (1993)

5. Bergweiler, W, Eremenko, A: On the singularities of the inverse to a meromorphic function of finite order. Rev Mat Iberoamericana. 11, 355-373 (1995)

6. Chen, HH, Fang, ML: On the value distribution of flf. Sci China Ser A. 38, 789-798 (1995)

7. Fang, ML, Hua, XH: Entire functions that share one value. J Nanjing Univ Math Biquarterly. 13(1), 44-48 (1996)

8. Yang, CC, Hua, XH: Uniqueness and value-sharing of meromorphic functions. Ann Acad Sci Fenn Math. 22(2), 395-406 (1997)

9. Fang, ML, Qiu, HL: Meromorphic functions that share fixed-points. J Math Anal Appl. 268, 426-439 (2002). doi:10.1006/ jmaa.2000.7270

10. Dyavanal, RS: Uniqueness and value-sharing of differential polynomials of meromorphic functions. J Math Anal Appl. 374, 335-345 (2011). doi:10.1016/j.jmaa.2010.09.002

11. Dou, J, Qi, XG, Yang, LZ: Entire functions that share fixed-points. Bull Malays Math Sci Soc. 34(2), 355-367 (2011)

12. Fang, ML: Uniqueness and value-sharing of entire functions. Comput Math Appl. 44, 823-831 (2002). doi:10.1016/S0898$1221(02) 00194-3$

13. Fang, ML, Hong, W: A unicity theorem for entire functions concerning differential polynomials. Indian J Pure Appl Math. 32(9), 1343-1348 (2001)

14. Lahiri, I: Weighted value sharing and uniqueness of meromorphic functions. Complex Var. 46, 241-253 (2001). doi:10.1080/17476930108815411

15. Li, XM, Gao, L: Meromorphic functions sharing a nonzero polynomial CM. Bull Korean Math Soc. 47(2), 319-339 (2010). doi:10.4134/BKMS.2010.47.2.319

16. Lin, WC, Yi, HX: Uniqueness theorems for meromorphic function concerning fixed-points. Complex Var. 49(11), 793-806 (2004). doi:10.1080/02781070412331298624

17. Lin, WC, Yi, HX: Uniqueness theorems for meromorphic function. Indian J Pure Appl Math. 35, 121-132 (2004)

18. Nevanlinna, R: Einige Eindeutigkeitssätze in der Theorie der Meromorphen Funktionen. Acta Math. 48, 367-391 (1926). doi:10.1007/BF02565342

19. Shen, SH, Lin, WC: Uniqueness of meromorphic functions. Complex Var Elliptic Equ. 52(5), 411-424 (2007). doi:10.1080/ 17476930601159467

20. Xu, JF, Lü, F, Yi, HX: Fixed-points and uniqueness of meromorphic functions. Comput Math Appl. 59, 9-17 (2010). doi:10.1016/j.camwa.2009.07.024

21. Zhang, JL: Uniqueness theorems for entire functions concerning fixed-points. Comput Math Appl. 56, 3079-3087 (2008). doi:10.1016/j.camwa.2008.07.006

22. Zhang, TD, Lü, WR: Uniqueness theorems on meromorphic functions sharing one value. Comput Math Appl. 55, 2981-2992 (2008). doi:10.1016/j.camwa.2007.11.029

23. Zhang, $\mathrm{XB}, \mathrm{Xu}$, JF: Uniqueness of meromorphic functions sharing a small function and its applications. Comput Math Appl. 61, 722-730 (2011). doi:10.1016/..camwa.2010.12.022

24. Zhang, XY, Lin, WC: Uniqueness and value-sharing of entire functions. J Math Anal Appl. 343, 938-950 (2008). doi:10.1016/j.jmaa.2008.02.011

25. Xu, JF, Yi, HX, Zhang, ZL: Some inequalities of differential polynomials. Math Inequal Appl. 12, 99-113 (2009)

26. Zhang, ZL, Li, W: Picard exceptional values for two class differential polynomials. Acta Math Sinica.34, 828-835

27. Köhler, L: Meromorphic functions shareing zeros and poles and also some of their derivatives sharing zeros. Complex Var. 11, 39-48 (1989). doi:10.1080/17476938908814322

doi:10.1186/1029-242X-2012-100

Cite this article as: Cao and Zhang: Uniqueness of meromorphic functions sharing two values. Journal of Inequalities and Applications 2012 2012:100. 